\title{
DIAGNÓSTICO DA ATIVIDADE PESQUEIRA NO ESPÍRITO SANTO, BRASIL: UM ESTUDO SOBRE O SEGMENTO DE PEIXARIAS
}

\author{
Keuwy Sousa ROCHA ${ }^{1}$; Caroline Tedesco SANTOS ${ }^{1}$; Rodrigo Randow de FREITAS ${ }^{1 *}$ \\ ${ }^{1}$ Universidade Federal do Espírito Santo (UFES). Centro Universitário Norte do Espírito Santo \\ (CEUNES). Departamento de Engenharia e Tecnologias (DETEC). \\ *e-mail: rodrigo.r.freitas@ufes.br
}

Recebido em: 22/03/2018

\begin{abstract}
Resumo - O consumo de peixes tem se elevado nas últimas quatro décadas e a tendência é que siga o ritmo de aumento anual. Além disso, o peixe continua a ser um dos produtos alimentares mais comercializados no mundo. A cadeia produtiva do pescado possui um importante papel entre as atividades econômicas do país, pois assume função importante na geração de emprego e renda para a população e na oferta de alimentos, não apenas para a população local. Com isso, para atender a esse crescimento da demanda, conhecer os stakeholders e os elos da cadeia produtiva se torna essencial para que a cadeia de suprimentos suporte esse crescimento de forma sustentável. Assim, o presente artigo buscou levantar questões relativas à atividade de pesca praticada, analisando o market share de um dos elos da cadeia produtiva, as peixarias, além de levantar problemas que dificultam o crescimento do comércio nesses estabelecimentos. Dentre eles estão a falta de pescado, infraestrutura deficiente e falta de fábrica de gelo. Por ser um diagnóstico inicial dessas empresas no estado, mais estudos são necessários, com os objetivos de mitigar os problemas encontrados e ampliar os fatores positivos.

Palavras-chave: pesca, cadeia produtiva, consumo pesqueiro, stakeholders
\end{abstract}

\section{DIAGNOSIS OF fISHERY ACTIVITY IN ESPÍRITO SANTO, BRAZIL: A STUDY ON THE SEGMENT OF FISH STORES}

Abstract - Fish consumption has risen over the past four decades and is likely to continue to increase annually. In addition, fish remains one of the most commercialized food products in the world. The fishing productive chain plays an important role among the economic activities of the country, since it assumes an important role in generating employment and income for the population and food supply, rather than only for the local population. In order to meet this growth in demand, it is essential to know the stakeholders and the links in the production chain, for the supply chain to support such growth in a sustainable way. Thus, this article seeks to raise questions about the fishing activity practiced, analyzing the market share of one of the links in the chain of production, the fish stores, in addition to raising problems that hinder the growth of trade in these establishments. Among them are the lack of fish, poor infrastructure and lack of ice production. As it is an initial diagnosis of these companies in the state, more studies will be needed to mitigate the problems encountered and to amplify the positive factors.

Key-words: fishing, productive chain, fishing consumption, stakeholders 


\section{INTRODUÇÃO}

Produtos pesqueiros têm sido utilizados como alimento desde os homens primitivos. Fato observado por meio dos fragmentos de ossos e pedras e de desenhos gravados em cavernas, indicando que esses homens eram caçadores e pescadores (Santos, 1977). O pescado consumido não é decorrente apenas de pesca extrativista, subsistência ou industrial, mas também dos cultivos, atividade praticada há milhares de anos. Por exemplo, registros arqueológicos apontam sua existência no Egito desde o século XX a.C. e o primeiro livro sobre métodos de criação de carpas foi escrito pelos chineses em 500 a.C. (Schmidt, 1994).

Quando analisado como alimento e fonte de nutrientes, segundo o órgão da Organização das Nações Unidas voltado para agricultura e alimentação (FAO, 2014), o pescado é uma excelente fonte de proteína e nutrientes essenciais. Cita-se que uma porção de 150 gramas de peixe pode prover entre 50 e $60 \%$ da proteína diária que um adulto precisa. Dessa forma, essa fonte proteica pode representar um componente crucial em países com baixos índices de ingestão total de proteínas. Ainda, de acordo com esse estudo, o consumo por pessoa deve aumentar até 2030, assim como a produção total de peixes, que deverá atingir 187 milhões de toneladas, quase 45 milhões de toneladas a mais que em 2008.

Peixes marinhos fornecem 80 milhões de toneladas de alimento rico em proteína e micronutrientes para consumo humano anualmente. Além disso, contribuem em US\$230 bilhões para a economia global (Sumaila, Cheung, Lam, Pauly \& Herrick, 2011), e a produção global de peixes tem potencial de crescimento de 3,4\% em média, quando consideradas diferentes regiões espalhadas pelo globo (Blanchard et al., 2012).

No Brasil, a atividade pesqueira gera um produto interno bruto nacional de R\$ 5 bilhões e proporciona 3,5 milhões de empregos diretos e indiretos. Segundo o ranking mundial de 2010, o país ocupava a décima sétima posição na produção de pescados em cativeiro e a décima nona na produção total de pescados; e em 2014 os brasileiros já consumiam uma média de quase 10 quilos por habitante anualmente (Pinheiro, 2014).

Já quando tratamos especificamente do estado do Espírito Santo, segundo Martins \& Doxsey (2006), a produção anual de peixes era de aproximadamente 22.095 toneladas. Reitera-se que em 2011 esse valor era de 26.816 toneladas. Além disso, Teixeira, Lima, Boechat, Rodrigues \& Freitas (2012) mostram que a atividade é considerada organizada e planejada nas regiões sul e central do estado, onde existem empresas estruturadas para exercer a produção em larga escala.

A frota pesqueira do Espírito Santo é considerada a maior frota de pesca oceânica do país, segundo o Ministério da Pesca e Aquicultura (MPA), por contabilizar um contingente de 2.486 barcos motorizados e 11.517 pescadores ativos. Calcula-se que em vários pontos desse extenso 
litoral, parte significativa da população ainda viva exclusiva ou indiretamente da pesca artesanal (Oliveira, Souza \& Valle, 2007).

Em meio à importância dessa atividade econômica do país, como geração de emprego e renda, a carência na infraestrutura é um grande entrave e os stakeholders (público estratégico, parte interessada ou interveniente) podem estar mais conscientes dos problemas locais de uma comunidade, fornecendo informações valiosas para os grupos interessados (Voyer, Gladstone \& Goodall, 2012). Quanto a essa análise dos stakeholders, ela originou-se em meados de 1960 como uma ferramenta de suporte nos processos de gestão. É uma abordagem para entender um sistema através da identificação de atores-chave e interesses específicos (Rosso, Bottero, Pomarico, La Ferlita \& Comino, 2014). Esses atores não são apenas os grupos de interesse, mas também pessoas e organizações que são envolvidas direta ou indiretamente e são afetados pelo cenário em processo (Brugha \& Varvasovszky, 2000).

Dessa forma, baseado nas informações apresentadas, o pescado apresenta-se como um dos produtos alimentícios mais comercializados no mundo e com crescimento constante. Para atender ao aumento da demanda, conhecer os stakeholders, os elos de sua cadeia produtiva e o market share que a atividade possui se torna essencial para que a cadeia de suprimentos suporte esse crescimento de forma sustentável. Assim, o presente artigo buscou levantar questões relativas a fatores qualitativos (problemas dessas empresas na comercialização do pescado) e quantitativos (quantidade e preço do pescado comercializado pelas peixarias) acerca da atividade de pesca praticada, analisando um dos elos da cadeia produtiva, as peixarias, além de levantar problemas que dificultam o crescimento do comércio nesses estabelecimentos.

\section{MATERIAIS E MÉTODOS}

O presente estudo foi realizado em peixarias de municípios do estado do Espírito Santo, Brasil, durante 10 meses, entre 20/03/2017 e 12/12/2017, sendo que a área de estudo foi dividida em três regiões: norte (compreende os municípios de São Mateus, Linhares e Conceição da Barra), centro (inclui Aracruz, Guarapari e Vila Velha) e sul (corresponde a Anchieta e Itapemirim) (Figura 1). 


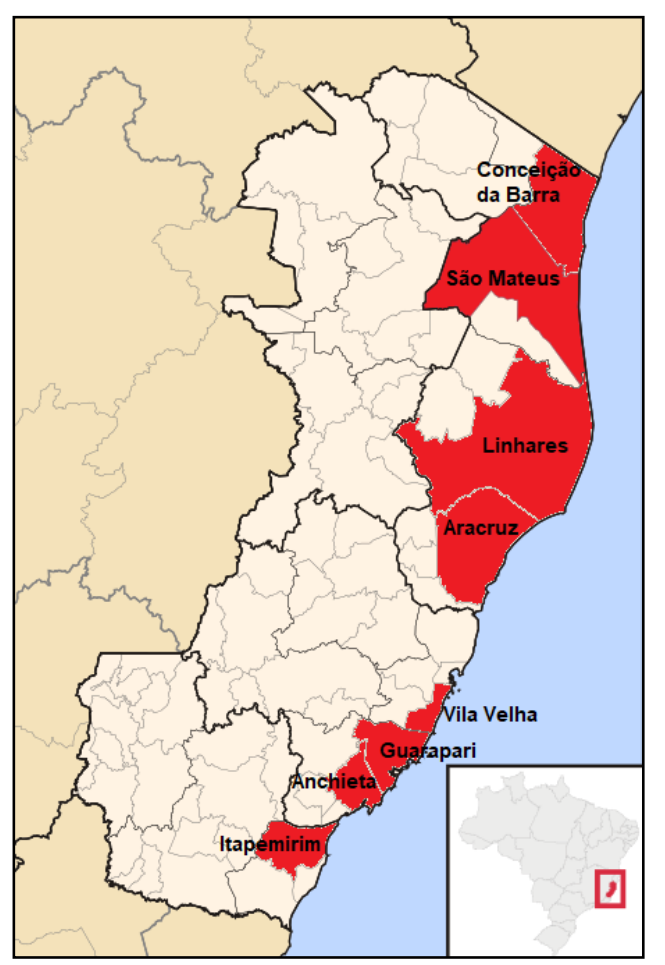

Figura 1. Mapa da localização geográfica das cidades da área de estudo.

\section{METODOLOGIA APLICADA}

A coleta de dados utilizou técnicas de observação direta intensiva, que é realizada por meio da técnica de observação e entrevista. A observação foi feita por um pesquisador fora da realidade estudada e foi sistemática, ou seja, estruturada, planejada e controlada, o que consiste em ver, ouvir e examinar os fatos e fenômenos que se deseja estudar (Kauark, Manhães \& Medeiros, 2010).

O método de amostragem utilizado no estudo foi o snowball sampling, também conhecido como método bola de neve ou cadeia de informantes. Foram utilizados informantes-chave, conhecidos como sementes, para que fossem identificadas pessoas com perfil adequado para a pesquisa. Com isso, o quadro da amostragem cresceu a cada entrevista (Vinuto, 2014). Logo, a amostragem adotada foi a não probabilística por acessibilidade, sendo utilizados os elementos que estavam acessíveis para a coleta dos dados, buscando eficiência, representatividade e fidedignidade das características dos dados coletados (Gil, 2002).

No levantamento de dados foi definido que o alvo do estudo seria um elo específico da cadeia produtiva do pescado local, as peixarias, levantando questões relativas a fatores qualitativos (problemas dessas empresas na comercialização do pescado) e quantitativos (quantidade e preço do pescado comercializado pelas peixarias) acerca da atividade praticada.

O tamanho da amostra, ou o número de respondentes necessários para o levantamento do estudo, depende do quão grande é a população e qual a confiabilidade desejada para os resultados 
obtidos (Cay \& Uyan, 2013). Dessa forma, objetivando o dimensionamento do tamanho da amostra, neste trabalho foi adotada a expressão apresentada na Equação 1 (Dupont \& Plummer, 1990).

$$
n=\frac{Z^{2} \cdot(x / n) \cdot[1-(x / n)] \cdot N}{(N-1) \cdot e^{2}+Z^{2} \cdot(x / n) \cdot[1-(x / n)]}
$$

Em que:

$\mathrm{n}=$ tamanho da amostra;

$\mathrm{N}=$ tamanho da população;

$e=$ erro amostral;

$x / n=$ proporção estimada do item pesquisado na amostra $(\%)$; e

$\mathrm{Z}=$ valor da abscissa da curva normal associada ao nível de confiança.

Dentre os municípios litorâneos, as peixarias distribuídas pelo estado são aproximadamente 269 (Nardoto et al., 2005), sendo assim, foi preciso obter 71 respostas para que o questionário tivesse validade.

Foram realizadas análises de agrupamento hierárquico por similaridade (cluster) e escalonamento multidimensional não paramétrico. Foi utilizado o software Primer (Plymouth Routines In Multivariate Ecological Research), versão 6, para melhor visualizar as relações de proximidade entre os subíndices, indicadores e alternativas, considerando o grau de importância fornecido pelos entrevistados e as informações quantitativas coletadas.

Para o método de classificação dos stakeholders, foi utilizada a abordagem apresentada por Mendelow (1981) chamada mapeamento de stakeholders, utilizando a matriz de poder/interesse (Figura 2).

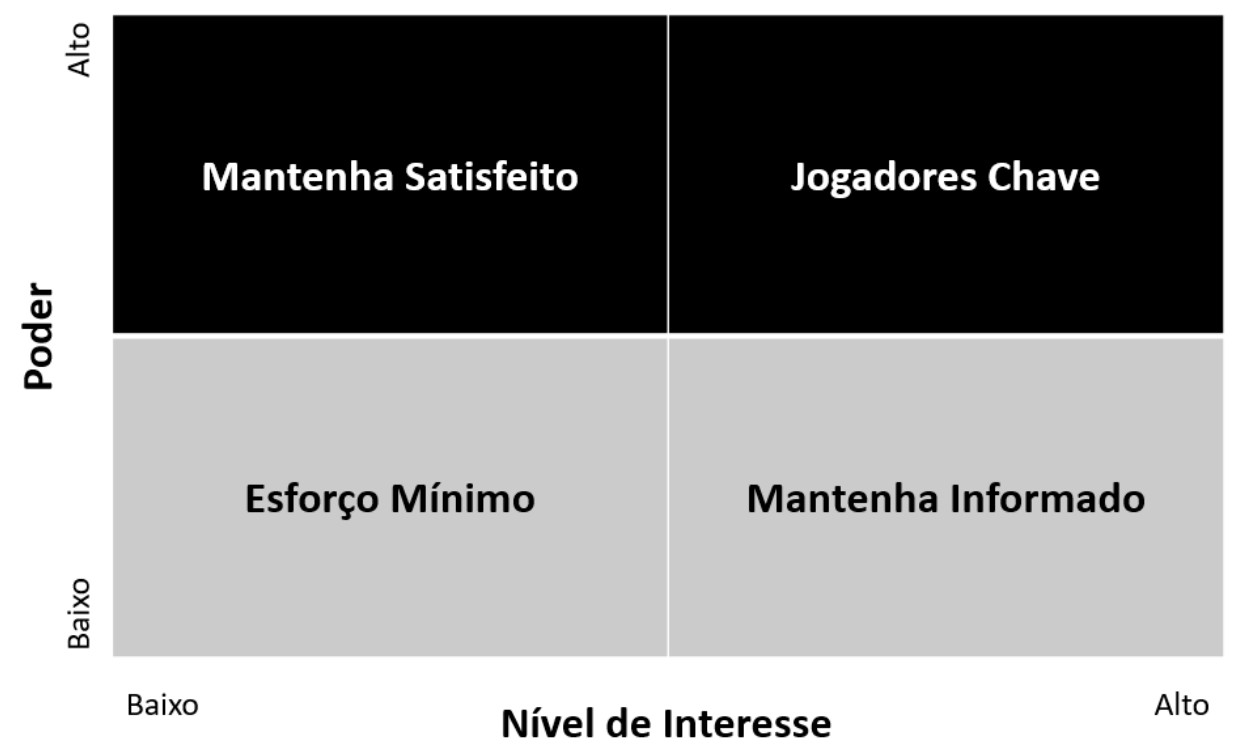

Figura 2. Matriz poder/interesse. 
Fonte: adaptado de Mendelow (1981).

Para essa identificação e análise, foi elaborada uma listagem das principais categorias de atores, os quais influenciam a atuação da organização ou são influenciados por ela. Para isso, foram utilizados como base os atores identificados por Martins, Rodrigues, Ribeiro \& Freitas (2015), sendo divididos em internos (pessoas ou entidades mais próximas da organização) e externos (que possuem algum tipo de interesse e que, de alguma forma, possam influenciar o negócio).

Baseado nisso, os stakeholders foram classificados como internos (1 - pescadores, 2 restaurantes, 3 - clientes) e externos (4 - governo, 5 - prefeituras, 6 - Instituto Capixaba de Pesquisa, Assistência Técnica e Extensão Rural - INCAPER, 7 - MPA, 8 - Instituto Brasileiro do Meio Ambiente e dos Recursos Naturais Renováveis - IBAMA, 9 - Instituto Estadual de Meio Ambiente - IEMA, 10 - agentes facilitadores, como os bancos, entre outras instituições que apoiam a pesca).

Para análise dos atores-chave na matriz de poder/interesse, cada um foi avaliado separadamente em relação ao poder e interesse que possui na cadeia produtiva. Para isso, foi utilizada pontuação de 0 a 10: 0 indica baixo poder/interesse e 10 indica alto poder/interesse. A pontuação foi definida pelo tempo deste projeto, baseado no conhecimento adquirido ao ler sobre o tema.

Depois que as pontuações dos atores foram definidas, calculou-se a porcentagem de importância que cada um possui na cadeia produtiva, baseada na metodologia apresentada por Rosso, Bottero, Pomarico, La Ferlita \& Comino (2014). Para o stakeholder 3, os clientes, foi definida a pontuação 9 de poder e 8 de interesse. Esses valores foram multiplicados para identificar a nota de importância, que foi 72. Baseado na soma de todas as notas de importância, foi definida a porcentagem de importância do stakeholder, 24,9\%.

Com base nos atores-chave 3 , clientes, foi identificado o market share dos produtos vendidos pelas peixarias. $\mathrm{O}$ market share corresponde à participação de mercado de uma empresa ou grupo dentro do seu segmento de atuação (Kotler, 1999). Dessa forma, foram verificados os estados em que as empresas comercializam seus produtos, ou seja, onde possuem participação de mercado.

Por fim, quanto à metodologia adotada para integração e análise dos dados, com o intuito de proporcionar uma melhor visualização dos resultados obtidos, foi elaborada uma matriz SWOT (Strenghts/forças, Weaknesses/fraquezas, Opportunities/oportunidades e Threats/ameaças). Isso propiciou uma melhor definição das potencialidades e possíveis fraquezas intrínsecas ao tema. Com isso, foram identificados os pontos fortes, pontos fracos, fraquezas e oportunidades, com o objetivo de analisar de forma macro o ambiente corporativo da proposta (Chiavenato \& Sapiro, 2003).

\section{ReSUltados E Discussão}


A cadeia produtiva possui alguns segmentos, até que o produto chegue ao consumidor final. O primeiro envolve o suprimento de bens e insumos necessários ao desenvolvimento da atividade, onde está incluída a produção de embarcações, os motores e petrechos de pesca e os insumos básicos. A base da cadeia, o segmento de produção de pescado, envolve as empresas de pesca industrial e os pescadores artesanais. No próximo elo está o processo de comercialização, no qual acontecem as funções agregadoras de valor e utilidades de posse, forma, tempo e espaço ao produto, que conduzem até o mercado consumidor (Santos, 2005).

Os segmentos de transporte e distribuição envolvem os agentes responsáveis por levar o produto, através de diferentes canais de comercialização, até o consumidor final. O consumidor final adquire o pescado em feiras livres, peixarias, supermercados ou sob a forma de pratos prontos em restaurantes e hotéis (Santos, 2005).

Assim, pode-se observar que após a captura, o destino dos pescados no estado são as peixarias, restaurantes e intermediários. O presente estudo buscou estudar as peixarias dos 14 municípios litorâneos do Espírito Santo (Oliveira, Souza \& Valle, 2007). As peixarias de Fundão, Serra, Vitória, Piúma, Marataízes e Presidente Kennedy não foram entrevistadas por problemas de logística e orçamento, mas o tamanho da amostra é confiável diante da população de estudo no estado.

Assim, 77 peixarias foram entrevistadas e foi considerada a quantidade (kg) comercializada, como apresentado na Tabela 1. Entre as principais espécies identificadas estão: pescadinha (Macrodon ancylodon), badejo (Mycteroperca spp.), corvina (Micropogonias spp.), cação (tubarão), camarão (Penaeidae), peroá (Balistes capriscus), dourado (Coryphaena hippurus), pargo (Lutjanus purpureus) e garoupa (Epinephelus spp.), que correspondem aproximadamente a $83 \%$ da quantidade pescada.

Tabela 1. Representação da quantidade e do preço médio do pescado vendido nos estabelecimentos em cada cidade e região do Espírito Santo, na estação de safra e final de safra (médias).

\begin{tabular}{cccc}
\hline Cidade & Estação & $\begin{array}{c}\text { Quantidade } \\
\text { (kg/estabelecimento) }\end{array}$ & Preço (R\$) do kg \\
& Safra & $2.011,50$ & 11,66 \\
\cline { 2 - 4 } Linhares & $\begin{array}{c}\text { Final de } \\
\text { safra }\end{array}$ & 560,60 & 12,16 \\
& Safra & 354,00 & 12,25 \\
\hline \multirow{2}{*}{ São Mateus } & $\begin{array}{c}\text { Final de } \\
\text { safra }\end{array}$ & 88,70 & 12,63 \\
\hline $\begin{array}{c}\text { Conceição da } \\
\text { Barra }\end{array}$ & Safra & $1.434,75$ & 11,25 \\
\cline { 2 - 4 } & Final de & $2.297,96$ & 12,00 \\
\hline
\end{tabular}




\begin{tabular}{|c|c|c|c|}
\hline & safra & & \\
\hline \multirow[b]{2}{*}{ Aracruz } & Safra & $7.554,00$ & 13,90 \\
\hline & $\begin{array}{c}\text { Final de } \\
\text { safra }\end{array}$ & - & 15,58 \\
\hline \multirow[b]{2}{*}{ Guarapari } & Safra & $6.060,63$ & 13,81 \\
\hline & $\begin{array}{c}\text { Final de } \\
\text { safra }\end{array}$ & $1.161,26$ & 14,92 \\
\hline \multirow[b]{2}{*}{ Vila Velha } & Safra & 822,00 & 17,04 \\
\hline & $\begin{array}{c}\text { Final de } \\
\text { safra }\end{array}$ & 563,75 & 19,56 \\
\hline \multirow[b]{2}{*}{ Anchieta } & Safra & $6.904,50$ & 9,33 \\
\hline & $\begin{array}{c}\text { Final de } \\
\text { safra }\end{array}$ & $4.416,00$ & 12,59 \\
\hline \multirow[b]{2}{*}{ Itapemerim } & Safra & $189.245,50$ & 9,02 \\
\hline & $\begin{array}{c}\text { Final de } \\
\text { safra }\end{array}$ & $54.507,00$ & 10,73 \\
\hline
\end{tabular}

Foram realizadas análises de similaridade entre as cidades estudadas. O agrupamento hierárquico interliga as amostras realizando associações e produz um dendrograma, fazendo com que tais amostras se agrupem. Quanto menor a distância entre os pontos, maior será a semelhança entre as amostras (Moita Neto \& Moita, 1998).

Com base nisso, observa-se que no período de safra os estabelecimentos possuem similaridade, devido à proximidade entre as cidades analisadas, exceto para o município de Itapemirim. Isso se deve à presença de uma empresa de pesca no município, que explica a quantidade elevada de peixes que são comercializados, superando as demais. Essa diferença é perceptível quando analisamos o número de funcionários fixos, um total de 84 . Os outros estabelecimentos estudados na cidade, que são peixarias, possuem no máximo três funcionários fixos.

Os peixes negociados nos municípios estudados são resultado da pesca artesanal, diferentemente da pesca industrial realizada em Itapemirim, que corresponde a $78 \%$ da produção das oito cidades. Diante disso, observou-se a disparidade que existe entre as duas atividades.

Os outros municípios possuem similaridade, quando analisada a quantidade média por estabelecimento. As quantidades comercializadas nesses locais são bem próximas, como os municípios de Guarapari, Aracruz e Anchieta, além de Linhares e Conceição da Barra, que possuem similaridade acima de $80 \%$. São Mateus possui uma similaridade menor porque foi registrada uma quantidade inferior de peixes comercializados no município. Dentre os problemas apresentados pelos entrevistados em São Mateus, que possuem influência na redução das quantidades comercializadas, está a falta de fábrica de gelo, a infraestrutura deficiente e a concorrência, pois o produto é processado manualmente, o que aumenta o custo produtivo (Nardoto et al., 2005). 
A operação mais crítica na manipulação do pescado é conseguir um resfriamento rápido. A conservação de peixe no gelo tem sido usada para aumentar o tempo de vida comercial desse alimento (Gaspar Jr., Vieira \& Tapia, 1997; Lira, Pereira \& Athayde, 2001). Assim, a falta de uma fábrica de gelo aumenta os custos para conservação dos peixes, o que explica as menores quantidades comercializadas, pois uma maior quantidade demandaria mais gelo.

Esses dados corroboram os estudos realizados por Dias (2010), que afirma que a falta de investimento por parte das instituições e a precária infraestrutura, principalmente em relação às condições higiênico-sanitárias das peixarias, levam à redução da procura pelos clientes.

Quando analisada a quantidade por espécie, a similaridade do município de Linhares com Conceição da Barra e Vila Velha foi menor. Enquanto nas peixarias de Linhares foram encontradas 17 espécies, sendo as mais representativas: pescadinha (958,3 kg/peixaria), sarda (138,3 kg/peixaria), cação (79,17 kg/peixaria), caçari (Cathorops spixii; 237,7 kg/peixaria) e pescada (Macrodon spp; 181,7 kg/peixaria), em Conceição da Barra foram identificadas 22 espécies, mas a pescadinha (126,7 kg/peixaria) mostrou ser a mais comercializada. Quando se considera Vila Velha, a comercialização foi representativa para cação (130 kg/peixaria) e camarão 7 barbas (Xiphopenaeus kroyeri; $225 \mathrm{~kg} /$ peixaria).

Ao analisar o período de final de safra, observou-se que em Aracruz não houve produção. Isso aconteceu pois não foi possível obter os valores de quantidade de peixes por estabelecimento durante esse período. O município não foi retirado do estudo com o intuito de mostrar a dificuldade de se obter dados dessa atividade. Reis, Julio, Gomes \& Freitas (2017) constataram que as comunidades de seu estudo, constituídas por pescadores artesanais e tradicionais de Pedra D’Água, Guriri e Barra Nova, localizados em São Mateus, ES, Brasil, não possuem nenhum tipo de coleta e análises sistemáticas de dados em relação aos processos de produção, beneficiamento e comercialização.

Quando se buscou analisar por regiões estudadas (norte, centro e sul), a similaridade foi pequena. É difícil encontrar similaridades porque a quantidade de pescado comercializado nesse período sofre grande redução. Algumas espécies comercializadas na safra deixam de ser no final da safra. Além disso, não existe um padrão nessa redução. Por exemplo, no município de Linhares, a pescadinha (958,3 kg/peixaria) é muito mais comercializada que o vermelho (83,3 kg/peixaria), mas no final de safra, o vermelho é mais vendido que a pescadinha.

Essa diferença entre safra e final de safra acontece devido à redução da oferta, comum na atividade pesqueira, como mostram Braga, Espírito-Santo, Silva, Giarrizzo \& Castro (2006) sobre a comercialização de pescado em Bragança, Pará, e em Manaus, Amazonas, também descrita por Parente \& Batista (2005). Como há disparidade na oferta do pescado na safra e no final de safra, há 
também alteração no preço, sendo maior no final de safra, por causa da menor oferta do produto (Figura 3).

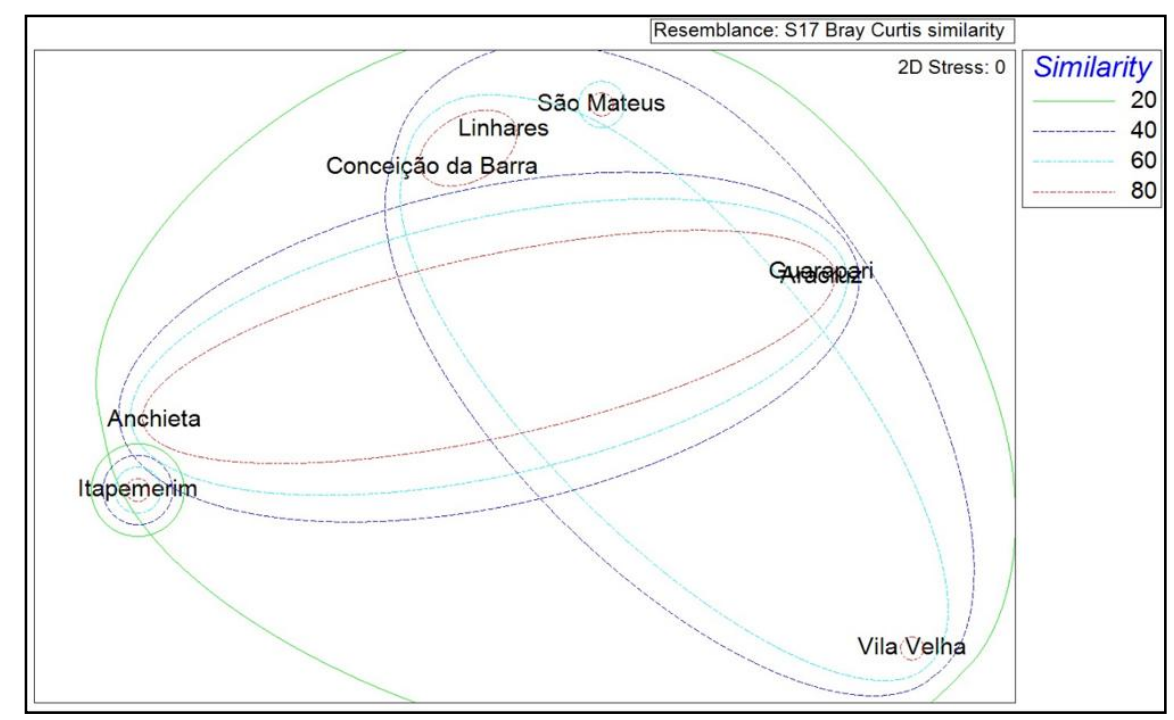

Figura 3. Similaridade com relação ao preço na estação de safra nos estabelecimentos localizados nas cidades estudadas.

Além da quantidade comercializada nessas localidades, foi analisada a variável preço entre elas. É possível observar que o preço é bem similar entre os municípios de cada região. Por exemplo, Conceição da Barra, Linhares e São Mateus, que compõem a região norte, possuem preços médios por produto muito próximos. O mesmo acontece nas regiões centro e sul, exceto para Vila Velha, onde foram encontrados preços muito superiores aos outros municípios.

Essa similaridade acontece porque o sucesso de uma empresa não está associado apenas à formação do preço em si, mas também à sua relação com clientes e concorrentes (Nagle \& Holden, 2003). Como esses municípios estão na mesma região, é compreensível que os preços sigam um padrão entre eles.

Essa característica compreende um mercado de concorrência perfeita, no qual os compradores e vendedores comercializam um produto uniforme, com as mesmas características (Bruni \& Famá, 2003). Nesse mercado as empresas não ultrapassam os preços estipulados pela concorrência, para que os clientes não deixem de comprar seus produtos (Hansen \& Mowen, 2001).

O fato de Vila Velha possuir menor similaridade com os outros municípios pode ser explicado pelo fato de que as espécies comercializadas possuem maior valor de venda, como camarão, cação e dourado.

\section{ANÁLISE DOS STAKEHOLDERS}

A partir da metodologia apresentada, a matriz de poder/interesse foi elaborada, como pode ser observado na Figura 4. A partir dela foi calculada a porcentagem de importância dos stakeholders. 
Os pescadores possuem importância de 34,6\%; valor esse que é compreensível, pois como a matriz apresenta, eles estão entre os atores-chave da cadeia, assim como os clientes (24,9\%). Ambos possuem tal representatividade por causa da dependência que as peixarias possuem, pois os pescadores capturam o produto a ser vendido e os clientes são o destino final da comercialização. Sem esses dois atores a cadeia produtiva se torna deficiente.

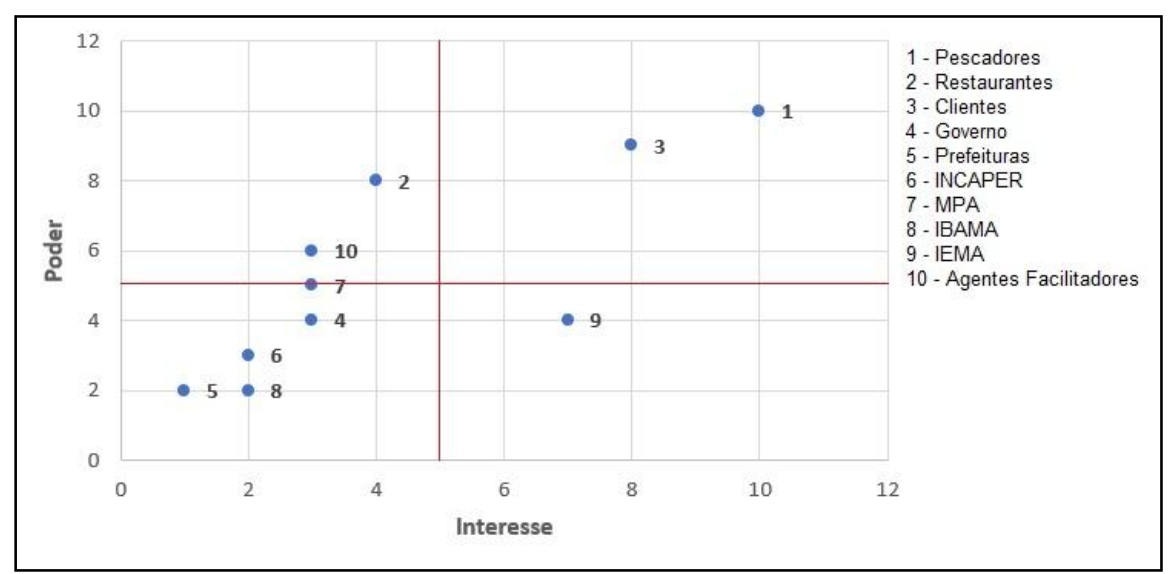

Figura 4. Matriz poder/interesse.

Os restaurantes possuem grau de importância de 11,1\%. Apesar de alguns restaurantes serem clientes de peixarias, foi observado no estudo que essa participação é irrelevante para serem considerados clientes, pois geralmente compram o produto de forma direta (Figura 4).

Com relação aos demais stakeholders, governo (4,2\%), prefeituras $(0,7 \%)$, INCAPER $(2,1 \%)$, MPA (5,2\%), IBAMA (1,4\%), IEMA $(9,7 \%)$ e agentes facilitadores $(6,2 \%)$, esses possuem uma participação menor, pois não estão envolvidos diretamente na produção ou comercialização do produto. Esses atores são estruturas de sustentação da cadeia, de forma indireta. Toda essa cadeia é influenciada pelos ambientes institucionais e organizacionais que envolvem órgãos do governo e outras instituições relacionadas à governança ou coordenação da cadeia produtiva. Esses atores são responsáveis pelo estímulo e pela regulação das atividades produtivas (Santos, 2005).

Quando analisamos o market share, ou seja, a participação de mercado, a comercialização com outros estados é pequena, sendo mais representativa em Minas Gerais e em menor quantidade no Rio de Janeiro, Bahia, Ceará e Rio Grande de Sul. Assim, a participação das peixarias no mercado está direcionada para os clientes do Espírito Santo, mas há um potencial de crescimento para os estados citados.

A menor participação de mercado do pescado nos outros estados pode estar atrelada à falta de infraestrutura das peixarias, como já citado no decorrer do texto. Sem infraestrutura adequada, o 
atendimento a demandas interestaduais não se torna viável, dificultando a expansão do negócio. Além disso, a falta de fábricas de gelo, como em São Mateus, dificulta ainda mais esse comércio.

Além dos problemas apresentados, outro ponto foi um dos mais relatados pelos donos ou funcionários das peixarias: a falta de pescado. Com base nisso, esse foi identificado como um dos problemas principais, que afeta diretamente a cadeia produtiva e a zona de influência das peixarias. A falta de pescado também é uma dificuldade encontrada em comunidades em Santarém, Pará (Zacardi, Saraiva \& Vaz, 2017), por exemplo.

As causas para esse problema podem ser diversas, dentre elas a pesca predatória, que tem reduzido a quantidade de peixes. Esse é um problema que já foi relatado por pescadores em pesquisa realizada por Knox \& Trigueiro (2015) no litoral do Espírito Santo. Essa situação é encontrada em Vitória, Anchieta, Regência e Linhares.

Diante desses problemas e para buscar um melhor entendimento sobre a cadeia produtiva, foi elaborada uma matriz SWOT (Figura 5). Analisando as forças do ambiente interno, a pesca é realizada por comunidades de pescadores que possuem tradição na atividade. Essa experiência permite melhor identificação das espécies de maior demanda, o que é positivo para as peixarias. Além disso, as peixarias estão localizadas nos municípios litorâneos, próximos dos pescadores, o que reduz custos relacionados ao transporte do produto até esses estabelecimentos.

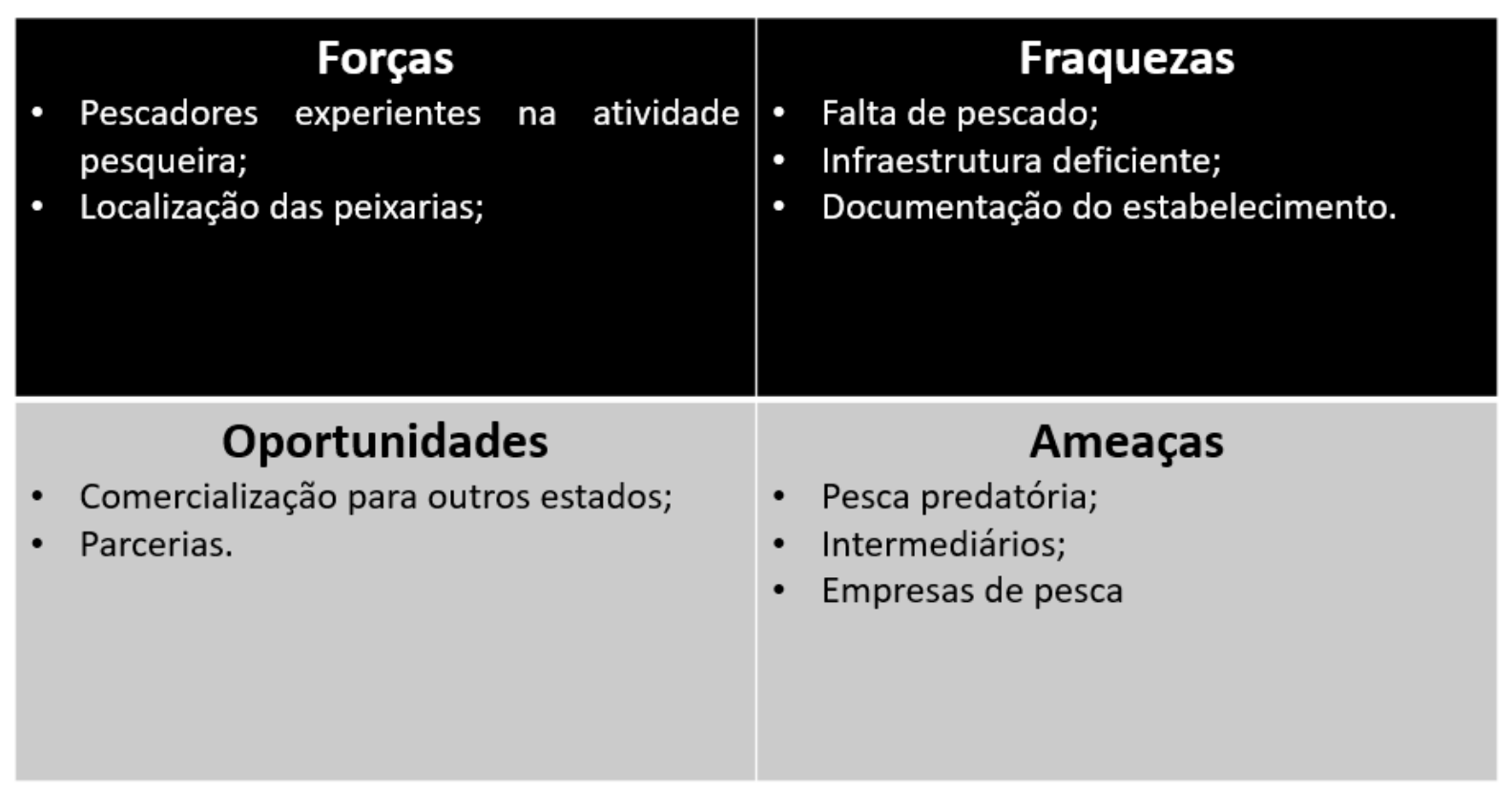

Figura 5. Matriz SWOT (Strenghts/forças, Weaknesses/fraquezas, Opportunities/oportunidades e Threats/ameaças) das peixarias do estado do Espírito Santo.

Com relação às fraquezas, a falta de pescado, problema relatado anteriormente, impacta diretamente a cadeia produtiva, pois o pescado é o produto principal comercializado. Além disso, 
problemas de infraestrutura dos estabelecimentos podem ocasionar perda de qualidade do produto. E, ainda, algumas peixarias não possuem documentação, funcionando ilegalmente, por causa da burocracia, segundo os questionários.

Analisando o ambiente externo, observou-se a oportunidade de crescimento na comercialização para os outros estados, pois as peixarias possuem poucos clientes fora do Espírito Santo. Além disso, parcerias podem surgir, com órgãos responsáveis pela pesca ou universidades, para a melhoria da cadeia, pois é uma atividade com tradição no estado.

Uma ameaça à atividade é a pesca predatória ou excessiva, que segundo os entrevistados tem causado redução do número de peixes disponíveis, resultando na falta de pescado, relatado anteriormente como uma fraqueza. Ainda, a existência de intermediários que vendem o pescado para as peixarias é ponto negativo para esses estabelecimentos, pois são vendidos por valores mais altos, reduzindo o lucro das peixarias. Além do mais, a existência de empresas de pesca, como de Itapemirim, impacta na questão de quantidade e preço dos pescados com relação às peixarias.

\section{Conclusão}

A partir dos resultados obtidos, ficou evidente que os grupos da cadeia produtiva do Espírito Santo precisam de melhorias na estrutura de apoio à pesca. O comércio realizado por peixarias possui grandes oportunidades de crescimento, mas está estagnado diante dos problemas encontrados, como infraestrutura deficiente, falta de pescado e falta de fábricas de gelo, que inviabilizam o melhor atendimento das necessidades dos consumidores, isto é, o acesso a pescados com qualidade e preço acessível, e também a expansão da participação de mercado desses estabelecimentos.

Por meio da análise das áreas de pesca e market share, constatou-se que a cadeia produtiva da pesca do Espírito Santo possui forças e oportunidades, como o conhecimento dos pescadores e a possibilidade de expansão da comercialização para outros estados, de forma mais significativa. Mas a infraestrutura dessas peixarias não é satisfatória, gerando problemas de capacidade e qualidade do pescado. Além disso, a falta de fábricas de gelo, como em São Mateus, acarreta custos extras a esses estabelecimentos, dificultando seu crescimento.

$\mathrm{O}$ estudo foi importante para diagnosticar as oportunidades e forças que as peixarias possuem diante da atividade pesqueira, além das fraquezas e ameaças que são barreiras para o desenvolvimento dessas empresas. Mais estudos são necessários, com planos de ação para mitigar os problemas encontrados e ampliar os fatores positivos.

Portanto, se os fatores positivos forem potencializados, as fraquezas minimizadas e as ameaças transformadas em oportunidades, a gestão da cadeia produtiva poderá se tornar mais eficiente e competitiva não somente no âmbito estadual, como também no âmbito nacional. 


\section{REFERÊNCIAS}

BLANCHARD, J., JENNINGS, S., HOLMES, R., HARLE, J., MERINO, G., ALLEN, J. I., HOLT, J., DULVY, N. K., BARANGE, M. (2012). Potential consequences of climate change for primary production and fish production in large marine ecosystems. Philosophical Transactions B, 367(1605): 2979-2989. https://doi.org/10.1098/rstb.2012.0231

BRAGA, C. F., ESPÍRITO-SANTO, R. V., SILVA, B. B., GIARRIZZO, T., CASTRO, E. (2006). Considerações sobre a comercialização do pescado no município de Bragança - PA. Bol. Téc.-Cient. CEPNOR, 6(1): 105-120. http://doi.org/10.17080/1676-5664/btcc.v6n1p105-120

BRUGHA, R. \& VARVASOVSZKY, Z. (2000). Stakeholder analysis: a review. Health Policy Plann. 15(3): 239-246.

BRUNI, A. L. \& FAMÁ, R. (2003). Gestão de Custos e Formação de Preço. São Paulo: Atlas.

CAY, T. \& UYAN, M. (2013). Evaluation of reallocation criteria in land consolidation studies using the Analytic Hierarchy Process (AHP). Land Use Policy, 30(1): 541-548. http://dx.doi.org/10.1016/j.landusepol.2012.04.023

CHIAVENATO, I. \& SAPIRO, A. (2003). Planejamento estratégico: fundamentos e aplicações. Rio de Janeiro: Elsevier.

DIAS, R. C. J. (2010). Avaliação e comparação das condições higiênico-sanitárias dos talhos e peixarias abrangidos pelo PACE no município de Santarém [Dissertação de Mestrado]. Lisboa, Portugal: Universidade Técnica de Lisboa.

DUPONT, W. D. \& PLUMMER, W. D. (1990). Power and sample size calculations: a review and computer program. Controlled Clin. Trials, 11(2): 116-128.

FOOD AND AGRICULTURE ORGANIZATION OF THE UNITED NATIONS (FAO). (2014). The state of world fisheries and aquaculture. FAO. Acessado em 29 de novembro de $2017 \mathrm{em}$ http://www.fao.org/3/a-i3720e.pdf.

GASPAR JR., J., VIEIRA, R. \& TAPIA, M. (1997). Aspectos sanitários do pescado de origem de água doce e marinha, comercializado na feira de Gentilândia, Fortaleza, Ceará. Rev. Hig. Aliment., 11(51): 20-23.

GIL, A. C. (2002). Como elaborar projetos de pesquisa. São Paulo: Atlas.

HANSEN, D. R. \& MOWEN, M. M. (2001). Gestão de Custos: Contabilidade e Controle. São Paulo: Pioneira Thomson Learning.

KAUARK, F. S., MANHÃES, F. C. \& MEDEIROS, C. H. (2010). Metodologia da Pesquisa: Um guia prático. Itabuna: Via Litterarum.

KNOX, W. \& TRIGUEIRO, A. (2015). A pesca artesanal no litoral do ES. In: W. Knox, A. Trigueiro (Eds.). Saberes, narrativas e conflitos na pesca artesanal (pp. 17-50). Vitória: EDUFES.

KOTLER, P. (1999). Marketing para o século XXI: como criar, dominar e conquistar mercados. São Paulo: Atlas. 
LIRA, G. M., PEREIRA, W. D. \& ATHAYDE, A. H. (2001). Avaliação da qualidade de peixes comercializados na cidade de Maceió - AL. Rev. Hig. Aliment., 15(84): 67-74.

MARTINS, A. S. \& DOXSEY, J. R. (2006). Diagnóstico da pesca no litoral do estado do Espírito Santo. In: V. J. Isaac, A. S. Martins, M. Haimovici, J. M. Andriguetto Filho (Eds.). A pesca marinha e estuarina do Brasil no início do século XXI: recursos tecnologias, aspectos socioeconômicos e institucionais. Belém: Editora Universitária UFPA.

MARTINS, N. G., RODRIGUES, D. A., RIBEIRO, G. M. \& FREITAS, R. R. DE. (2015). Avaliação da atividade pesqueira numa comunidade de pescadores artesanais no Espírito Santo, Brasil. Rev. Ges. Costeira Integr., 15(2): 265-275. http://dx.doi.org/10.5894/rgci514

MENDELOW. (1981). Environmental Scanning - the Impact of the Stakeholder Concept. In: International Conference on Information Systems. Cambridge: Anais.

MOITA NETO, J. M. \& MOITA, G. C. (1998). Uma introdução à análise exploratória de dados multivariados. Química Nova, 21(4): 467-469.

NAGLE, T. T. \& HOLDEN, R. K. (2003). Estratégia e táticas de preço: Um guia para decisões lucrativas. São Paulo: Pearson Prentice Hall.

NARDoto, J. I. O., CENTODUCATTE, J. G., SANTOS NETO, F. E., SOUZA, A. C. C. DE, OLIVEIRA, J. C. DE, BARROSO, M. V., BARROSO, G. F. \& OLIVEIRA, L. A. A. G. (2005). Macrodiagnóstico da Pesca Marítima do Estado do Espírito Santo. Acessado em 12 de novembro de 2017 em https://www.yumpu.com/pt/document/view/50423146/macrodiagnostico-da-pescamaritima-do-estado-do-espirito-santo-sig.

OliveIRA, J. C., SOUZA, A. C. C., VALLE, J. DE B. (EDS.). (2007). Plano Estratégico de Desenvolvimento da Agricultura Capixaba. Novo PEDEAG 2007-2025. Vitória: Secretaria de Estado de Agricultura, Pecuária, Abastecimento e Pesca.

PARENTE, V. M. \& BATISTA, V. S. (2005). A organização do desembarque e o comércio de pescado na década de 1990 em Manaus, Amazonas. Acta Amaz., 35(3): 375-382. http://dx.doi.org/10.1590/S0044-59672005000300011

PINHEIRO, A. (2014). Potencial Brasileiro. $1^{\text {o }}$ Anuário Brasileiro da Pesca e Aquicultura. $\begin{array}{lllllll}\text { Acessado em } & 12 & \text { de } & \text { julho } & \text { de } & 2017 & \mathrm{em}\end{array}$ http://formsus.datasus.gov.br/novoimgarq/16061/2489520_218117.pdf.

REIS, G. A., JULIO, T. S., GOMES, V. A. DO P. \& FREITAS, R. R. DE. (2017). Utilização da Metodologia Dmaic em Comunidades Pesqueiras Artesanais no Sudeste do Brasil. Rev. Bras. Eng. Pesca, 10(1): 1-16. http://dx.doi.org/10.18817/repesca.v10i1.1115

ROSSO, M., BOTTERO, M., POMARICO, S., LA FERLITA, S. \& COMINO, E. (2014). Integrating multicriteria evaluation and stakeholders analysis for assessing hydropower projects. Energy Police, 67: 870-881. https://doi.org/10.1016/j.enpol.2013.12.007

SANTOS, E. (1977). Pesca e piscicultura. Belo Horizonte: Itatiaia.

SANTOS, M. A. S. DOS. (2005). A cadeia produtiva da pesca artesanal no Estado do Pará: estudo de caso no Nordeste Paraense. Amazônia: Ci. \& Desenv., 1(1): 61-81.

SCHMIDT, A. (1994). Piscicultura: a fonte divertida de proteínas. São Paulo: Ícone. 
SUMAILA, R. U., CHEUNG, W. W. L., LAM, V. W. Y., PAULY, D. \& HERRICK, S. (2011). Climate change impacts on the biophysics and economics of world fisheries. Nature Climate Change, 1: 449-456. https://doi.org/10.1038/nclimate1301

TEIXEIRA, J. B., LIMA, A. C., BOECHAT, F. P., RODRIGUES, R. L. \& FREITAS, R. R. (2012). Potencialidade social e econômica da pesca e maricultura no Estado do Espírito Santo, Brasil. Rev. Ges. Costeira Integr., 12(4): 569-575. http://dx.doi.org/10.5894/rgci372

VINUTO, J. (2014). A amostragem em Bola de Neve na pesquisa qualitativa: um debate em aberto. Temáticas, 22(44): 203-220.

VOYER, M., GLADSTONE, W. \& GOODALL, H. (2012). Methods of social assessment in Marine Protected Area planning: is public participation enough? Marine Policy, 36(2): 432-439. https://doi.org/10.1016/j.marpol.2011.08.002

ZACARDI, D. M., SARAIVA, M. L. \& VAZ, E. M. (2017). Caracterização da Pesca Artesanal Praticada nos Lagos Mapiri e Papucu às Margens do Rio Tapajós, Santarém, Pará. Rev. Bras. Eng. Pesca, 10(1): 31-43. http://dx.doi.org/10.18817/repesca.v10i1.1158 